\title{
Analyzing Nurses' Responsibilities in the Neonatal Intensive Care Unit Using SHERPA and SPAR-H Techniques
}

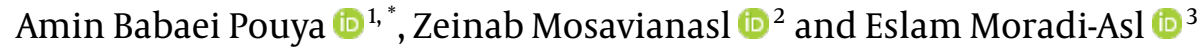 \\ ${ }^{1}$ Department of Occupational Health Engineering, School of Health, Ardabil University of Medical Sciences, Ardabil, Iran \\ ${ }^{2}$ Department of Occupational Health and Safety Engineering, School of Health, Larestan University of Medical Sciences, Larestan, Iran \\ ${ }^{3}$ Department Public Health, School of Public Health, Ardabil University of Medical Sciences, Ardabil, Iran \\ "Corresponding author: Ardabil University of Medical Sciences, Ardabil, Iran. Tel: +98-4533513775, Fax: +98-4533512004, Email: amiin.pouya@yahoo.com
}

Received 2018 July 08; Revised 2019 March 11; Accepted 2019 March 16.

\begin{abstract}
Background: Neonatal intensive care unit (NICU) is a critical unit in terms of nursing care with a high risk of error incidence. Objectives: This research aimed at determining the type of nursing task mistake, the risk level of the nurse's duties, and assessing the probability of human error in the duties of the nurse.

Methods: This research was a case study. The location of NICU research was Alavi and Bouali Hospitals in Ardabil University of Medical Sciences in 2018. The study was performed using HTA, SHERPA and SPAR-H methods.

Results: Having analyzed the occupational hierarchy, 17 main tasks and 35 subtasks were identified and studied in NICUs. Among 156 error cases, $43.59 \%$ were action errors (highest frequency) and 8.97\% were selection errors (lowest frequency). The most common errors in terms of the type of task and probability of errors were "air and respiratory ventilation", "thermal and respiratory monitoring", "examination, evaluation and control of the newborn's pain”, and "administering drugs".

Conclusions: The factors affecting performance such as time, psychological and physical stress, workload, work complexity, mental effort, experience and education, instruction, ergonomics principles, work planning, safety culture, management policy, and organizational support had the greatest impact on the probability of nurse errors.
\end{abstract}

Keywords: Intensive Care Unit, Human Errors, Nurse

\section{Background}

Studies regarding the field of human error in treatment process showed that human error has mostly occurred by physicians, nurses and other health system staff; but the promising point for studying the errors in the diagnosis and treatment process was the possibility to identify and prevent the incidence of its consequences. According to the studies, $70 \%$ of medical errors are preventable (1-3). Almost any human health expert causes damage to the patient by human error. In the industrial sector, it has been reported that human error occurs, thus the systems are designed to detect errors and prevent their incidence. However, several large studies have confirmed the occurrence of medical errors in clinical activities with possible patient damages. However, the problems caused by these errors have long been underestimated (4). The neonatal intensive care unit (NICU) is a critical unit in terms of nursing care with a high risk of error incidence (5). The advances made in NICU have increased the survival rate of premature infants, but these infants need treatment measures and complicated medical care (6). Preserving and improving the health of infants as a vulnerable group in healthcare services is of great importance. Premature infants experience their critical growth period in the NICU (7). There are several methods for doing job analysis. One of the methods mostly used to identify human errors, is the hierarchical task analysis (HTA), which was described by Stanton in 2006 (8). The HTA divides all job tasks into a hierarchical process into a set of sub-tasks, and presents it in the form of a figure or table. The HTA refers to the presentation of a detailed image of a nurse's activity in a system and in the execution of tasks that it carries. The most important advantage of this method is to decompose tasks to smaller ones, in order to predict the errors better. In the study by Lane et al., HTA was used for the drug prescription model, and then systematic human error reduction and prediction approach were used to predict possible errors (9). Another study by Sarker et al. found that HTA could be used to evaluate the technical skills specific 
to surgeonsand residents (10). The second most commonly used method for identifying human error is the systematic human error reduction and prediction approach (SHERPA) method. SHERPA was created by Embrey in 1986 (11). This technique examined action error, retrieval error, checking error, selection error, and communication error. In the Khandan et al. study, SHERPA has been used as an approach for managing errors in nurses' health and treatment and improving patient safety (12). In another study by Kermani et al. nurses' errors in the emergency department were identified using the SHERPA technique (13). The third method was standardized plant analysis risk-human (SPAR-H) reliability analysis. This method was first used by Blackman et al. (14) in 1994, as a simple method for calculating human error probabilities. The objective of the performance shaping factor (PSF), was to determine the nominal human error probability (NHEPs), and calculating the ultimate human error probability by considering the degree of dependency between tasks. In the study of Mohammadfam et al., the SPAR-H method was used to assess quantitative human error in nursing tasks (15). In another study by Tanha et al., SPAR-H method was used to assess the nurses' mistakes in the emergency department of delivery in a hospital affiliated to Tehran University of Medical Sciences (16). This research has been conducted to determine the type of nursing task mistake, the risk level of the nurses' duties, and assess the probability of human error in the duties of the nurses.

Various studies have been conducted regarding the evaluation of nurses' errors. However, no study has been conducted using SHERPA and SPAR-H techniques simultaneously for evaluating nurses' errors.

Among evaluation techniques of human errors, SHERPA technique has been selected for recognizing the type of error and presenting ways for reducing the errors. Moreover, SPAR-H technique was chosen for recognizing performance shaping factors and presenting the last possibility of the error.

\section{Objectives}

The current study aimed at quantitatively and qualitatively analyzing the neonatal intensive care unit nurses' duties by HTA, SHERPA and SPAR-H methods to identify tasks with the highest probability of incidence of errors. Having identified the factors affecting the occurrence of errors, the results were delivered to treatment managers and supervisors of the department to provide the necessary interventions such as management, ergonomics and train- ing to control the errors.

\section{Methods}

This research was a case study. The location of NICU research was Alavi and Bouali hospitals in the Ardabil University of Medical Sciences in 2018. The study was performed using HTA, SHERPA and SPAR-H methods.

The checklists of the study were completed by two health experts through having interviews with nurses and documentary observations.

The project was carried out with the consent of the unit's officials and the findings of the plan were made available to the authorities to improve the working conditions.

The present study was conducted to determine the type of errors, description of errors, description of the consequences, determination of the level of risk, description of the corrective strategies, determining the factors affecting the performance, determining the error probability and determining the tasks with the highest probability of error. Prior to the implementation of the techniques, determining the jobs and tasks was critical.

\subsection{HTA Method}

The first step was to use the HTA method. There were several methods for job analysis. One of these methods, which was most often used to identify human errors, was the hierarchical task analysis method. An analysis of job tasks was done through the HTA method. The first step was to determine the critical tasks (8-10).

HTA phases:

- Phase 1: Determine job

- Phase 2: Determine task

- Phase 3: Determine sub-task

\subsection{SHERPA Method}

The second step was to use the SHERPA method (Table 1).

- Phase 1: Task classification: Each phase of task for classification of the error was considered as:

- Action: Setting the temperature, opening or closing key, filling the daily report notebook.

- Retrieval: Receiving information via monitor or instructions.

- Checking: Monitoring and following up a review process.

- Selection: Selecting a solution with higher authority orders. 


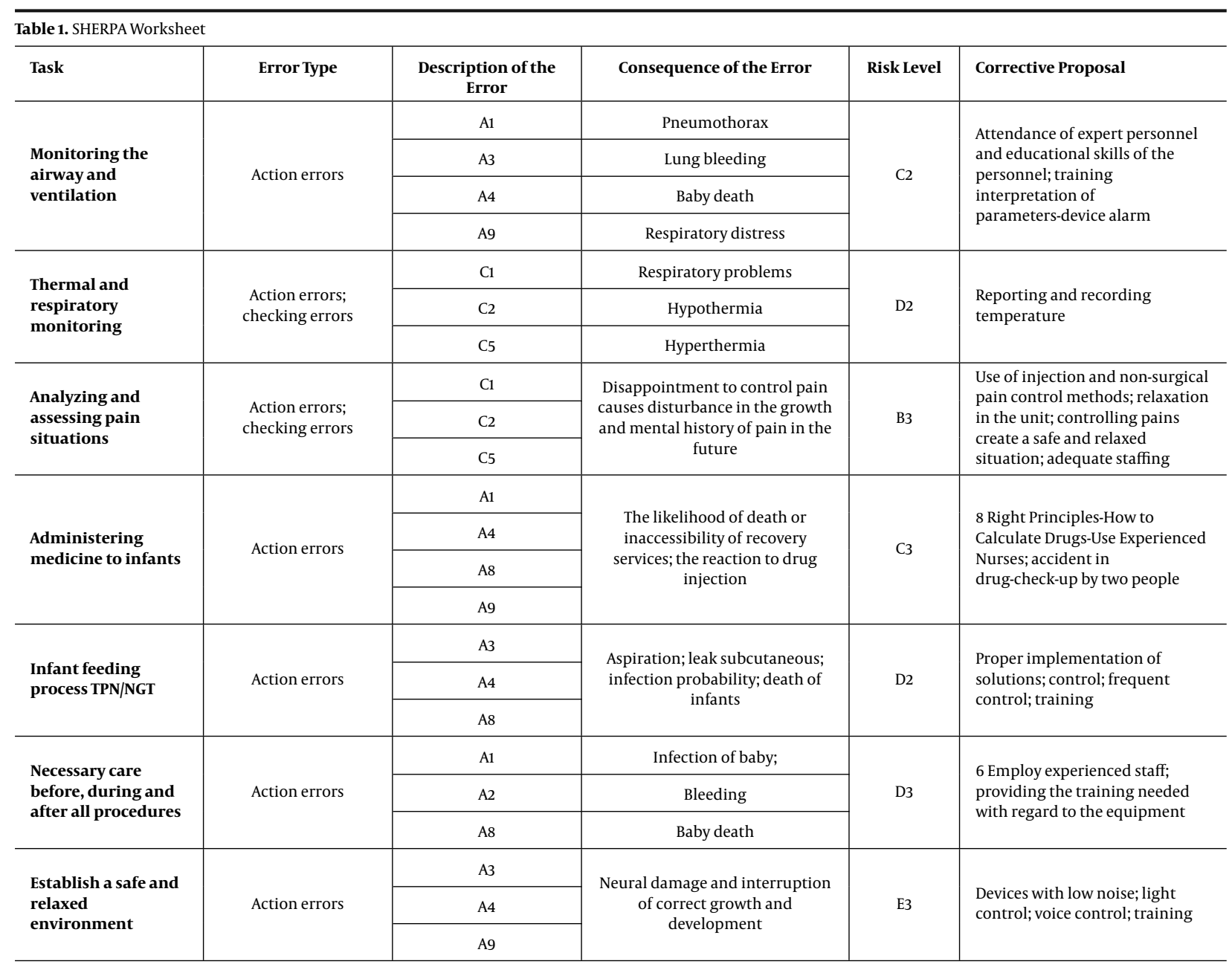

- Information communication: Negotiations with colleagues

- Phase 2: Human error identification: In this step, various human errors were examined.

- Action error: The action was not performed on time and properly.

- Retrieval error: Immediate action after the problem to return to normal mode.

- Checking error: Checking was not done on time and properly.

- Selection error: A phase, section, or item was mistakenly chosen or forgotten.

- Communication error: Information was not received correctly.

- Phase 3: Analysis: Studying and examining the consequences of each error

- Phase 4: Human risk assessment: Identified errors were assessed based on probability and severity.
- Phase 5: Remedy analysis: (equipment, training, instructions, management, and organization) efforts for reducing the error were suggested (11-13).

\subsection{SPAR-H Mthod}

The third step was to use the SPAR-H method (Table 2):

In step 3, to calculate the human error probability (HEP), the SPAR-H technique was used as follows (14-16):

- Phase 1: Dividing human tasks as recognition or action

There were similar questions and performance shaping factors for both diagnostic activities and practice, but the PSF coefficients were different. In some cases, when tasks were both diagnostic and functional, both cases were considered.

- Phase 2: Determining PSF score

At this phase, indicators affecting the performance of 
each identified task were determined. 8 indicators that affected the performance were:

1. Available time: (0.01, $0.1,1$ or 10 for recognition score) and $(0.01,0.1,1$ or 10 for action score $)$

2. Stress and stressors: (1, 2 or 5 for recognition score) and (1,2 or 5 for action score)

3. Experience and training: $(0.5,1$ or 10 for recognition score) and ( $0.5,1$ or 3 for action score)

4. Complexity: (0.1, 1, 2 or 5 for recognition score) and (1, 2 or 5 for action score)

5. Ergonomics: $(0.5,1,10$ or 50 for recognition score) and $(0.5,1,10$ or 50 for action score)

6. Procedures: $(0.5,1,5,20$ or 50 for recognition score) and $(1,5,20$ or 50 for action score)

7. Fitness for duty: ( 1 or 5 for recognition score) and ( 1 or 5 for action score)

8. Work processes: $(0.8,1$ or 2 for recognition score $)$ and ( $0.5,1$ or 5 for action score)

- Phase 3: Determining the NHEPs

At this phase, the nominal human error probability was determined and then multiplied by the factors affecting the performance $(\mathrm{PSF} 1 \times \ldots \times$ PSF8). NHEPs was 0.01 for recognition and 0.001 for action.

- Phase 4: Calculating HEP

PSF greater than or equal to 1 was considered as negative PSF. If there were less than 3 PSF cases detected in the number of PSF, the same number of steps, 3, was considered to be HEP. If there were three or more than three negative PSFs in the number of PSFs identified, the following formula was used to calculate the HEP.

HEP $=($ NHEP.PSF $) /$ NHEP. $($ PSF-1 $)+1$

- Phase 5: Calculating HEP with dependency

The amount of HEP was calculated by considering the dependence. Dependency in this case meant that the negative impact of a human error in the subsequent errors was reflected in the calculation of HEP.

In full dependence, the probability of error was considered to be 1.

In the high dependence the error probability was equal to $(1+$ HEP without dependency)/2

In the medium dependence, the probability of the error was equal to: $(1+6 \times$ HEP without dependency $) / 7$

In low dependencies, the error probability was equal to: $(1+19 \times$ HEP without dependency $) / 20$

In zero dependence, the error probability is equal to: HEP without dependency.

\section{Results}

\subsection{Findings of the HTA Method}

Having implemented the HTA method, 17 task and 35 sub-tasks were identified and studied for NICU nurses. The main identified tasks included the monitoring of the airway and ventilation, thermal and respiratory monitoring, analyzing and assessing pain situations of infants, administering medicine to infants, infant feeding process, recognition and treatment procedure healthcare, establish a safe and relaxed environment starting cardiopulmonary resuscitation for infant, phototherapy, sampling of arterial blood and interpreting the results, prevention of complications of arterial and venous sampling, tracheal intubation of infant, help in exchange transfusions of infant, help in catheterization of vein and umbilical artery, injecting surfactant care, Kangaroo care of infant, caring during transportation to dispatch counseling and para-clinical issues.

\subsection{Findings of the SHERPA Method}

Overall, the type of identified errors included 156 modes, $43.59 \%$ were associated with action error with the highest error type, and $8.97 \%$ was associated with the selection error, which was the lowest error type. Risk level assessment indicated that 3\% of identified errors had an undesirable risk level and $42.94 \%$ had an acceptable risk level, where, doing corrective interventions was necessary to reduce their risk level (Figure 1).

Figure 1 illustrats the number of recognized errors based on their type. For classifying the risks, a risk matrix has been utilized, whose risk possibility and intensity determined the risk level. In case the possibility and intensity of the risk was high, then one of the following: acceptable without revision, acceptable with revision, undesirable and unacceptable would be determined.

\subsection{Findings of SPAR-H Method}

The tasks identified with the highest probability of incidence of an error were: monitoring of the airway and ventilation, thermal and respiratory monitoring, analyzing and assessing pain situations, administering medicine to infants, infant feeding process, establish a safe and relaxed environment (Table 3). Identified factors having the greatest impact on the probability of human error included having available time less than required time to perform a task, high levels of psychological stress due to 


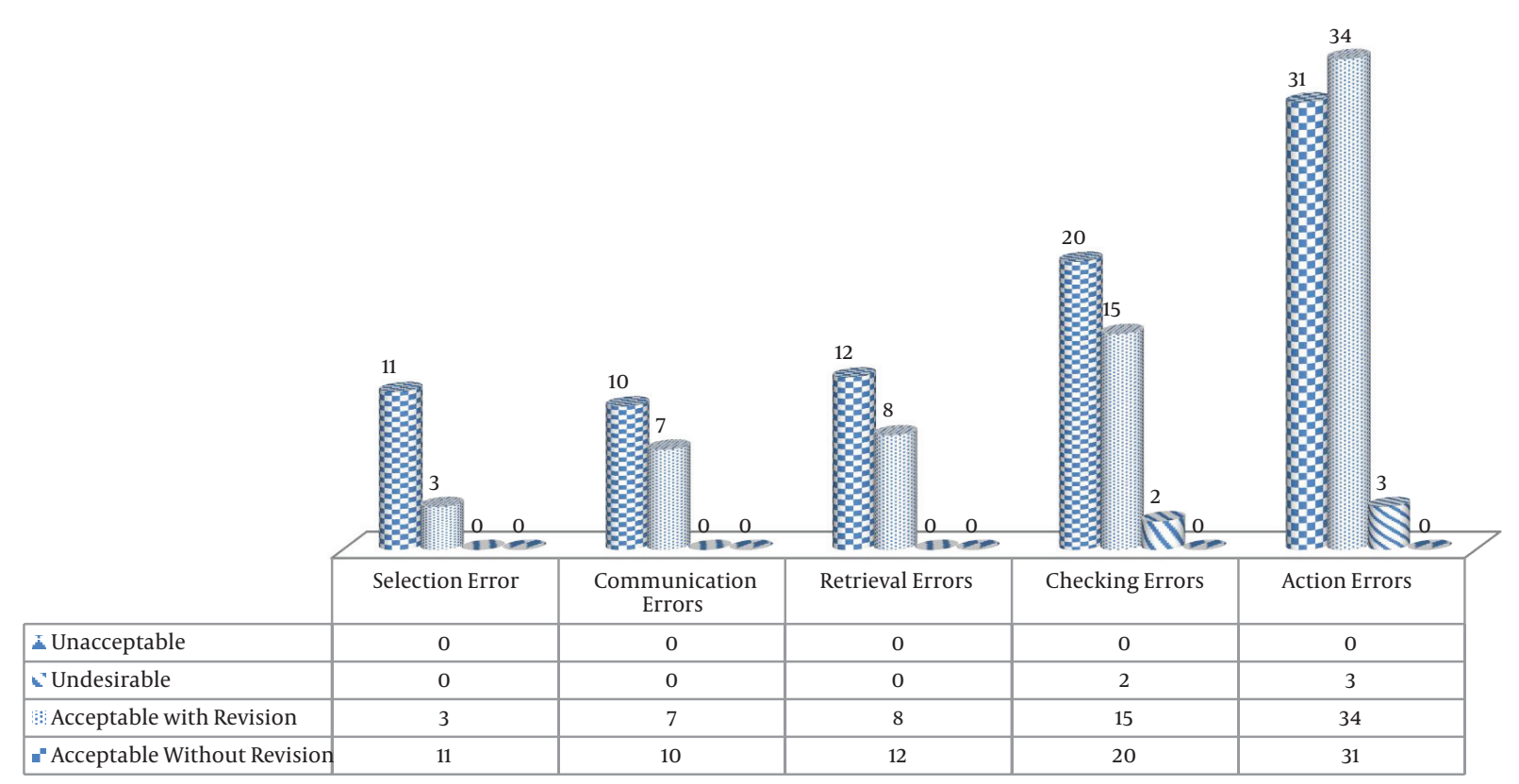

Figure 1. Level of risk, taking into account the type of error

excessive workload, physical stress due to fatigue, complexity of tasks such as performing multiple tasks, mental effort, further perceptual skills for doing tasks, education and experience to the task, instructions working in terms of quality and quantity, ergonomic monitoring equipment, appropriate arrangement of the display and controllers, task scheduling, safety culture, policy management, and organizational support.

\section{Discussion}

Among NICU nurses' responsibilities, 17 main responsibilities were recognized, through which 156 error moods were recognized, most of which belonged to the action error. Evaluating the risk level indicated that $3 \%$ of recognized errors had undesirable risk levels.

The recognized responsibilities that had affected the possibility of humane error occurrence included "the lack of time for doing the work", "high levels of psychological stress", "varieties and high loads of work", "physical stress due to fatigue" and "complicatedness of jobs", which were more important than the others.

In order to prevent mistakes in the care of the infant airway, the presence of expert personnel in shifts, training skills to personnel and training interpretation of $A B G$ arterial blood gases and interpreting parameters from $A B G$ before the creation of complications and attention to device alarms can be helpful. Events reported in the NICU on mechanical ventilation, blood products, intravascular lines, intravenous infusion and drug dose errors, showed the highest risk for patients in NICU (17). To prevent the occurrence of errors in the thermal and respiratory monitoring tasks, the reporting and recording of temperature should be done carefully. In order to prevent the occurrence of errors in the assessment and evaluation of the pain situations in infants, non-injectable and injectable pain control methods and establishing relaxation in the department and control of pain triggers could be used. In order to prevent mistakes in the task of administering medicine, to control errors from the correct principles of the patient medication, based on 8 Rights (correct patient, correct medication, correct time, correct use method, correct dose consumption, proper drug registration, correct administration of prescription, appropriate patient response to medication), training how to calculate medications periodically, using experienced nurses in all sections, careful medication and checking by two people. The study of frequency of diverse non-injectable medication errors among nurses of cardiac care units of Mazandaran province by Bagheri-Nesami et al. showed that using strategies such as increasing the knowledge of pharmacology of nurses and nursing students, providing standard conditions and improving communication between nurses and doctors, 


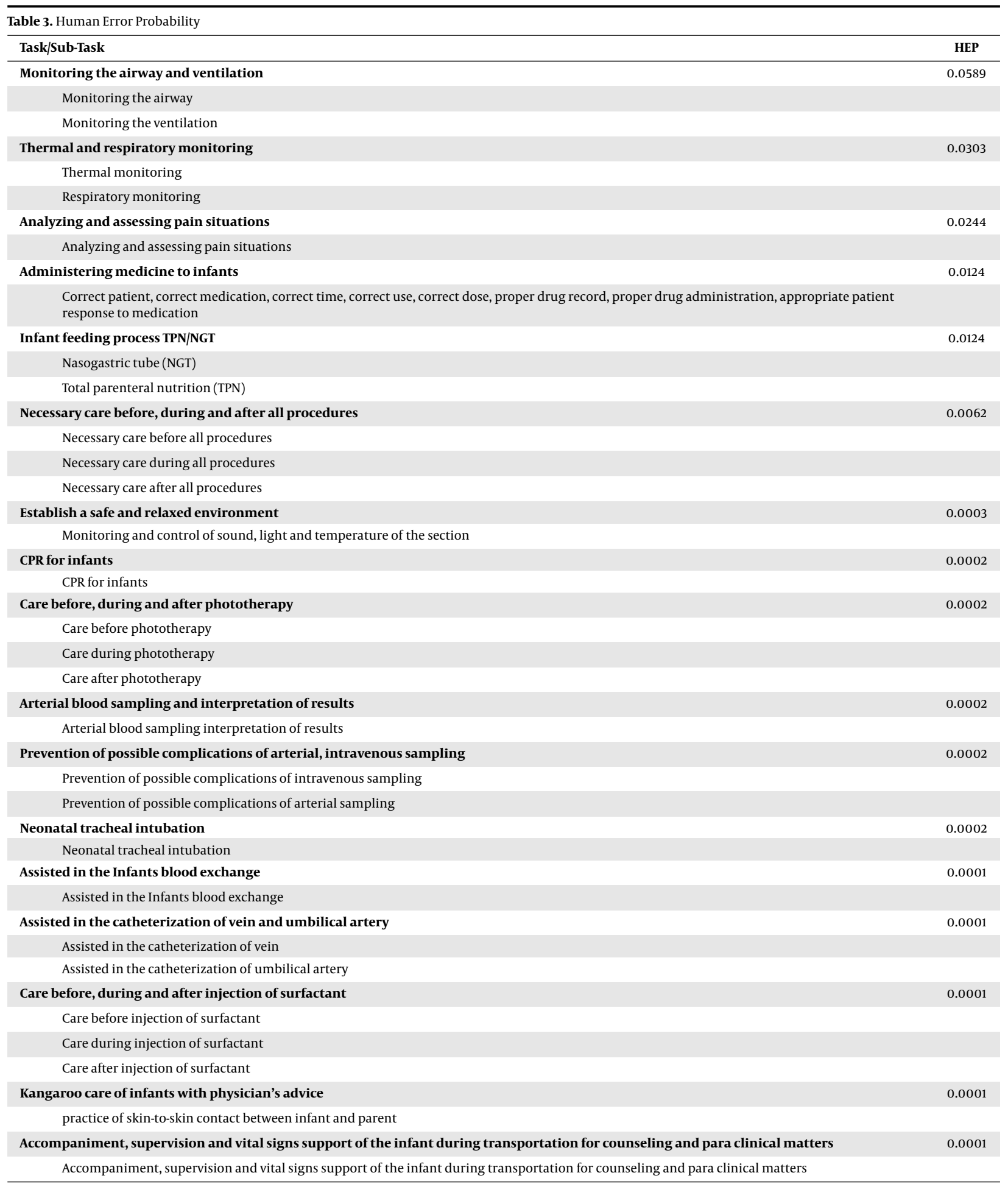

were recommended to limit the medication errors (18). The study of frequency and type of drug errors in the NICU of Yazd hospitals by Salmani and Hasanvand have shown that given the high frequency of errors, especially in the night shift, and knowing the involvement of imbalance of nurse to patient, it was necessary to further examine the causes 
and solutions of prevention of drug errors in future studies (19). The study of Alaee Karahroudy et al. about the nursing care associated with drug leakage in infants admitted to NICU was far from standard. This could be attributed to nurses' lack of awareness of the importance of proper care of drug leakage and its consequences, fault in training, lack of control of care by the officials and lack of facilities and equipment and shortage of nursing staff (20). Regarding the task of performing infant feeding including nasogastric tube (NGT), total parenteral nutrition (TPN) to track potential error of the correct interpretation of NGT and catheter, frequent control of shifts, and training the proper method, both should be considered. To prevent the occurrence of errors in the task of nursing care, before, during and after all diagnostic and treatment procedures, puncture, exchange of blood, umbilical catheter and peripherally inserted central catheter (PICC), the use of experienced personnel in the preparation of items needed before and after the procedure and training and retraining of nurses was necessary. In establishing a safe and relaxed environment to prevent neural stimulation, use of devices with low noise, control of light and sound in the department and training of personnel in this regard was necessary. Available time for doing work, workload, psychological and physical stress levels, complexity of tasks, mental effort, experience, training, instructions, ergonomic principles, work planning, safety culture, management policy and organizational support had the greatest impact on the probability of nurse error incidence. The shortage of nursing workforce in infants' intensive care unit had increased the number of working hours and led to the incidence of errors (21). The working balance of nurses in the hospital unit is important for the satisfaction and safety of nurses and patients. Workforce planning has to be done based on the variety and volume of work (22). Most incident reporting systems use a voluntary and unpunished approach to infant care. Voluntary reporting systems have identified many contributing factors in the incidence of errors (23). Hospital managers and nursing directors should make a lot of effort to promote group work, encourage the reporting of mistakes and improve patient safety (24). Interventions to identify nurses with mothers' expectations of quality and communication between nurse and mother seems to be necessary (25). Parents' training reduces their stress based on training needs. Having reduced the stress and enhanced the role of parents, the provision of high quality care during infant admission is ensured (26). Moreover, cyberspace training has been effective in increasing the psychological empowerment of nurses in infant inten- sive care units (27). By changing and modifying the educational process and educational planning through managers and nursing training authorities, it becomes possible to prevent the incidence of errors and repeating them in the future (28).

\subsection{Conclusions}

Increasing the number of nursing workforce, balancing workload of nurses, promoting group work, reducing mental and physical stress, compiling voluntary and unpunished incident reporting systems, increasing the quality of communication between nurses and mothers, strengthening parents' role, changing and modifying training processes, educating nurses and parents, establishing an environment with ergonomic principles and promoting a safety culture is suggested to reduce the risk of human error in the NICU section.

\section{Acknowledgments}

The authors of the paper are grateful to all the respectable staff of the NICU and the professional health personnel of the hospital that they have co-sponsored in this study with the authors of the paper.

\section{Footnotes}

Authors' Contribution: Eslam Moradiasl: data collection; Zeinab Mosavianasl: analysis; Amin Babaei Pouya: writing manuscript.

Conflict of Interests: The authors declare no conflict of interests.

Ethical Considerations: IR.ARUMS.REC.1397.022.

Funding/Support: This project supported by Ardabil University of Medical Science

Patient Consent: The project was carried out with the consent of the unit's officials.

\section{References}

1. Mazloumi A, Kermani A, Nasl Seraji J, Ghasem Zadeh F. [Identification and evaluation of human errors of physicians at emergency wardof an educational hospital in Semnan city using SHERPA technique]. Occup Med QJ. 2013;5(3):67-78. Persian.

2. Leape LL, Brennan TA, Laird N, Lawthers AG, Localio AR, Barnes BA, et al. The nature of adverse events in hospitalized patients. Results of the Harvard medical practice study II. NEngl JMed.1991;324(6):377-84. doi: 10.1056/NEJM199102073240605. [PubMed: 1824793].

3. Halbach J, Sullivan L. Medical errors and patient safety: A curriculum guide for teaching medical students and family practice residents. MedEdPORTAL Publications; 2005. doi:10.15766/mep_2374-8265.101. 
4. van der Schaaf TW. Medical applications of industrial safety science. BMJ Publishing Group Ltd; 2002.

5. Stokowski L. The link between RN staffing and infection in the NICU. The NICU: Safety in numbers. MedScape; 2013.

6. Kenner C, Sabuni F, Narenji F. Clinical nursing care of infant guideline. Arak, Iran: Research Office of Under-Secretary Arak University of Medical Sciences; 2001.

7. Cong X, Wu J, Vittner D, Xu W, Hussain N, Galvin S, et al. The impact of cumulative pain/stress on neurobehavioral development of preterm infants in the NICU. Early Hum Dev. 2017;108:9-16. doi: 10.1016/j.earlhumdev.2017.03.003. [PubMed: 28343092]. [PubMed Central: PMC5444300].

8. Stanton NA. Hierarchical task analysis: Developments, applications, and extensions. Appl Ergon. 2006;37(1):55-79. doi: 10.1016/j.apergo.2005.06.003. [PubMed: 16139236].

9. Lane R, Stanton NA, Harrison D. Applying hierarchical task analysis to medication administration errors. Appl Ergon. 2006;37(5):669-79. doi: 10.1016/j.apergo.2005.08.001. [PubMed: 16182230].

10. Sarker SK, Chang A, Albrani T, Vincent C. Constructing hierarchical task analysis in surgery. Surg Endosc. 2008;22(1):107-11. doi: 10.1007/s00464-007-9380-z. [PubMed: 17483993].

11. Embrey DE. SHERPA: A systematic human error reduction and prediction approach. Proceedings of the international topical meeting on advances in human factors in nuclear power systems. American Nuclear Society; 1986

12. Khandan M, Yusefi S, Sahranavard R, Koohpaei A. SHERPA technique as an approach to healthcare error management and patient safety improvement: A case study among nurses. Health Scope. 2016;6(2). doi: 10.5812/jhealthscope.37463.

13. Kermani AL, Mazloumi A, Kazemi Z. Identification of nurses' errors in the emergency ward, using SHERPA technique. Int J Occ Hygiene. 2016;8(1):54-61.

14. Blackman HS, Gertman DI, Boring RL. Human error quantification using performance shaping factors in the SPAR-H method. Proc Hum Fac Erg Soc Ann Meet. 2008;52(21):1733-7. doi: 10.1177/154193120805202109.

15. Mohammadfam I, Movafagh M, Bashirian S. Comparison of standardized plant analysis risk human reliability analysis (SPAR-H) and cognitive reliability error analysis methods (CREAM) in quantifying human error in nursing practice. Iran J Public Health. 2016;45(3):401-2. [PubMed: 27141509]. [PubMed Central: PMC4851761].

16. Tanha F, Mazloumi A, Faraji V, Kazemi Z, Shoghi M. [Evaluation of human errors using standardized plant analysis risk human reliability analysis technique among delivery emergency nurses in a hospital affiliated to Tehran University of Medical Sciences].J Hospital. 2015;14(3):57-66. Persian.
17. Snijders C, van Lingen RA, Klip H, Fetter WP, van der Schaaf TW, Molendijk HA, et al. Specialty-based, voluntary incident reporting in neonatal intensive care: Description of 4846 incident reports. Arch Dis Child Fetal Neonatal Ed. 2009;94(3):F210-5. doi: 10.1136/adc.2007.135020. [PubMed: 18838465].

18. Bagheri-Nesami M, Esmaeili R, Tajari M. [Frequency of non injectable medication administration errors in nurses of cardiac critical care units in Mazandaran province in 2014]. J Rafsanjan Univ Med Sci. 2016;15(2):151-64. Persian.

19. Salmani N, Hasanvand S. [Evaluation of the frequency and type of medication prescribing errors in the NICU of hospitals in Yazd] Hayat. 2016;21(4):53-64. Persian.

20. Alaee Karahroudy F, Naeimi N, Khanali Mojan L, Nasiri M. [The audit of nursing cares related to the treatment of extravasation in neonatal intensive care units]. Hayat. 2016;22(1):79-89. Persian.

21. Keels EL. Neonatal nurse and neonatal nurse practitioner fatigue. Newborn and Infant Nursing Reviews. 2016;16(3):168-72. doi 10.1053/j.nainr.2016.07.008.

22. Acar I, Butt SE. Modeling nurse-patient assignments considering patient acuity and travel distance metrics. J Biomed Inform. 2016;64:192206. doi: 10.1016/j.jbi.2016.10.006. [PubMed: 27742350].

23. Snijders C, van Lingen RA, Molendijk A, Fetter WP. Incidents and errors in neonatal intensive care: A review of the literature. Arch Dis Child Fetal Neonatal Ed. 2007;92(5):F391-8. doi: 10.1136/adc.2006.106419. [PubMed: 17376782]. [PubMed Central: PMC2675366].

24. Hwang JI, Ahn J. Teamwork and clinical error reporting among nurses in Korean hospitals. Asian Nurs Res (Korean Soc Nurs Sci). 2015;9(1):1420. doi: 10.1016/j.anr.2014.09.002. [PubMed: 25829205].

25. Salmani N, Dabirifard M, Maghsoudi Z, Dabirifard A, Karjo Z. [Comparing perception of nurse-mother communication between neonatal intensive care nurses and mothers of hospitalized neonates]. Hayat. 2016;22(3):291-9. Persian.

26. Ghavami SR, Borimnejad L, Seyedfatemi N, Haghani H. [The effect of parental role training on stress in the parents of hospitalized newborns in a neonatal intensive care unit]. Hayat. 2017;23(3):243-53. Persian.

27. Kadivar M, Seyedfatemi N, Zolfaghari M, Mehran A, Hosseinzadeh Z. [The impact of virtual-based education on nurses' psychological empowerment in the level II neonatal care unit]. Iran J Med Educ. 2017;17:102-15. Persian.

28. Kanani F, Valizadeh L, Mostafa Gharebaghi M, Asghari Jafarabadi M. [Types of nursing errors in gastric tube insertion procedure in preterm infants in neonatal intensive care unit, Sanandaj, 2013].J Urmia Nurs Midwifery Fac. 2016;13(11):953-63. Persian. 


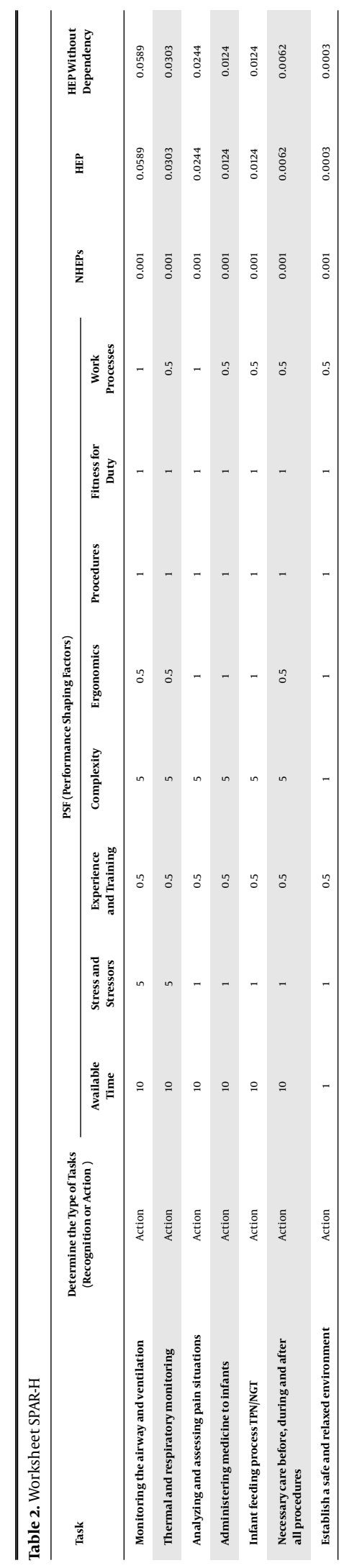

Shiraz E-Med J. 2019; 20(6):e81880. 